\title{
Resolução estética de mancha branca em esmalte através da associação de técnicas conservadoras à infiltração resinosa: Relato de caso
}

\author{
Esthetic resolution of enamel white spot lesion trough the association of conservative techniques to \\ resin infiltration: Case report
}

Resolución estética de lesión de mancha blanca del esmalte mediante la asociación de técnicas conservadoras a la infiltración resinosa: Relato de caso

Recebido: 10/06/2021 | Revisado: 18/06/2021 | Aceito: 19/06/2021 | Publicado: 04/07/2021

\author{
Bianca Fernandes Joaquim \\ ORCID: https://orcid.org/0000-0001-6821-1291 \\ Universidade Estadual do Norte do Paraná, Brasil \\ E-mail: biancajoaquim98@gmail.com \\ Thaina Mansano \\ ORCID: https://orcid.org/0000-0002-2513-3489 \\ Universidade Estadual do Norte do Paraná, Brasil \\ E-mail: thainamansano@hotmail.com \\ Sibelli Olivieri Parreiras \\ ORCID: https://orcid.org/0000-0003-1545-7663 \\ Universidade Estadual do Norte do Paraná, Brasil \\ E-mail: sibelliolivieri@hotmail.com \\ Mariana Vilela Sônego \\ ORCID: https://orcid.org/0000-0002-0498-4049 \\ Universidade Estadual do Norte do Paraná, Brasil \\ E-mail: mariana.sonego@uenp.edu.br
}

\begin{abstract}
Resumo
As lesões de mancha branca em esmalte são complicações clínicas comumente encontradas e causam desconforto nos pacientes por prejudicarem a estética. Uma alternativa vantajosa para tratar essas lesões é a infiltração resinosa, que garante a conservação da estrutura sadia do dente pela sua intervenção minimamente invasiva. Paciente com hipoplasia de esmalte nos dentes 12 e 13, teve como tratamento a associação do clareamento dental e microabrasão, seguida do o protocolo de infiltração resinosa $\left(\operatorname{Icon}^{\circledR}\right)$. Ao final dos procedimentos, foi constatada alteração cromática dos dentes, tornando a mancha imperceptível nos dentes da paciente. A principal vantagem da infiltração resinosa foi garantir a preservação de tecido dental saudável e reestabelecer a estética dental, mesmo em manchas profundas.
\end{abstract}

Palavras-chave: Lesão de mancha branca; Infiltração resinosa; Hipoplasia do esmalte dentário; Fluorose dentária.

\begin{abstract}
White spot lesions are a common clinical complication that affects dental esthetics and turn patients uncomfortable. An advantageous alternative to treat those lesions is the resin infiltration technique, that preserves healthy dental structure due its minimally invasive intervention. The patient had enamel hypoplasia on teeth 12 and 13 , that was treated with an in-office bleaching and microabrasion followed by resin infiltration $\left(\operatorname{Icon}^{\mathrm{TM}}\right)$. At the end of the procedure, it was noticed a color change, turning the spot unnoticeable on patient's teeth. The main advantage of resin infiltration is to guarantee teeth tissue preservation treatment while reestablishing esthetics, even when deep lesions are presented.
\end{abstract}

Keywords: White spot lesions; Resin infiltration; Dental enamel hypoplasia; Dental fluorosis.

\section{Resumen}

Las lesiones de manchas blancas son una complicación clínica común y causan malestar en los pacientes porque deterioran la estética. Una alternativa ventajosa para tratar estas lesiones es la infiltración resinosa, que garantiza la conservación de la estructura sana del diente por su acción mínimamente invasiva. Paciente con hipoplasia en los dientes 12 y 13 , recibió como tratamiento la asociación de la técnica de blanqueamiento dental y microabrasion con la posterior infiltración de resina (Icon®). Al final del procedimiento, se encontró alteración cromática de los dientes, haciendo la lesión enmascarada en los dientes del paciente. El tratamiento con infiltración de resina tiene como principal ventaja la preservación del tejido dental sano y poder restablecer la estética dental, mismo en lesiones profundas.

Palabras clave: Lesion de mancha blanca; Infiltración resinosa; Hipoplasia del esmalte; Fluorosis dental. 


\section{Introdução}

Manchas esbranquiçadas no esmalte podem ocorrer como consequência de danos pré ou pós eruptivos. Hipoplasia de esmalte, fluorose amelogênese imperfeita, e hipomineralização molar incisivo são exemplos de defeitos na formação do esmalte (Abdullah \& John, 2016; Attal et al., 2014; Denis et al., 2013). Já as lesões de mancha branca decorrente da cárie, são consideradas pós-eruptivas e podem atingir até 70\% em algumas populações (Sundararaj et al., 2015).

Apesar de o esmalte ser a estrutura de tecido mais mineralizado do corpo humano, formado por cristais de hidroxiapatita (96\%) e matriz orgânica (4\%) (Denis et al., 2013), defeitos em sua formação, como a hipomineralização, geram aumento de líquidos da matriz orgânica, alterando o nível de refração óptico do tecido. Ao invés de passar unidirecional por todo o tecido e ser refletido na junção amelodentinária, o feixe de luz é desviado na região da hipomineralização, o que faz com que a incidência de luz refletida aumente e se caracterize pelo aspecto branco percebido visualmente (Attal et al., 2014; Denis et al., 2013).

Enquanto a hipoplasia é uma alteração decorrente de traumas no capuz (Alverson et al., 2020; Khanna et al., 2020), a fluorose se caracteriza por uma alteração na fase de calcificação do desenvolvimento do esmalte em decorrência do consumo de flúor, sendo essa hipomineralização tecidual de forma generalizada ou pontual (Zotti et al., 2020) A amelogênese imperfeita é um defeito raro de formação tecidual na fase embrionária e dependo do grau em que ocorre, pode reduzir sua espessura, causar falhas ou produzir alterações cromáticas; normalmente acomete os incisivos e primeiros molares (Cavalheiro et al., 2020) As lesões de mancha branca cariosas são características na fase inicial da doença, lesões ativas são reconhecidas pelo aspecto rugoso e opaco, enquanto as lesões inativas se caracterizam por uma superfície lisa e brilhante (Gu et al., 2019).

Infelizmente, essas manchas opacas de cor "branco leitosas" localizam-se nos dentes anteriores e comprometem a aparência estética (Ciftci et al., 2018). Entretanto, diversas opções de tratamentos estão disponíveis para reverter a desmineralização do esmalte e/ou provocar mudança cromática. Os tratamentos mais invasivos consistem em restaurações dentárias diretas ou indiretas com resina composta ou cerâmica. São considerados invasivos pois exigem algum tipo de preparo e consequentemente, perda de estrutura dental. Dentre os tratamentos mais conservadores, encontram-se a micro abrasão, laser, o clareamento dentário, aplicação de verniz fluoretado, fosfato de cálcio amorfo e a infiltração resinosa. (Alverson et al., 2020; Brandão et al. 2020; Cavalheiro et al., 2020; Gençer \& Kirzioğlu, 2019; Giray et al., 2018; Gu et al., 2019; Kau et al., 2019, Peters et al., 2019; Sundfeld et al., 2019; Sundfeld et al., 2019; Yoo et al., 2019)

Apesar de recém desenvolvida, a técnica de infiltração resinosa tem demonstrado excelentes resultados estéticos (Alverson et al., 2020; Brignardello-Petersen, 2017; Cavalheiro et al., 2020; Marouane et al., 2018; Muñoz et al., 2013). A técnica consiste na infiltração de uma resina de baixa viscosidade (Icon ${ }^{\mathrm{TM}}$, DMG, Alemanha), que após a polimerização apresenta um índice de refração muito próximo ao esmalte sadio, mascarando a desmineralização e o aspecto opaco da lesão. A infiltração resinosa é de fácil manipulação e permite que o tratamento completo seja realizado em uma única sessão, sem trazer dor e desgaste ao esmalte dos dentes que recebem o tratamento. A infiltração resinosa pode ser realizada individualmente ou associada a outras técnicas invasivas ou não invasivas, a depender do diagnóstico da lesão e prognóstico do caso (Alverson et al., 2020; Marouane et al., 2018; Perdigão, 2020). O presente estudo relata a descrição de um caso clínico na resolução de manchas brancas nos dentes anteriores de uma paciente jovem.

\section{Metodologia}

Este trabalho descreve um relato de caso clínico realizado na clínica de atendimento odontológico da Universidade Estadual do Norte do Paraná (UENP), em que se realizou a correção estética de lesão branca no dente 12 e troca de restauração insatisfatória em resina composta do dente 13. A correção estética do elemento 12 (foco desse relato) se deu por meio da associação de técnicas conservadoras para preservação de estrutura dental sadia, foi utilizado o clareamento dental e micro abrasão, seguido pela infiltração resinosa. O relato de caso clínico foi a metodologia de escolha para a descrição do caso (Estrela, 
2018), que é caracterizado por ser abrangente tanto de planejamento, abordagens específicas, coleta e análise de dados, estabelecendo um estudo descritivo, com auxílio com auxílio de imagens, seguindo todos os princípios éticos (Pereira et al., 2018). Por se tratar de um relato de caso, não se faz necessário a submissão e aprovação do Comitê de Ética em Pesquisa, porém o paciente/responsável legal concorda e autoriza o uso das imagens, dados clínicos e radiográficos para fins educacionais através do Termo de Consentimento Livre e Esclarecido (TCLE) assinado (Fernandes, 2021). O embasamento científico do trabalho, foi feito através de buscas na literatura nas bases de dados Science Direct e PubMed, sem restrições de ano, utilizando as seguintes palavras-chave: "White Spot Lesions"; "Resin Infiltration”; “Dental Enamel Hypoplasia”; “Dental Fluorosis”.

\section{Relato de Caso}

A paciente, gênero feminino, 21 anos, compareceu à Clínica Odontológica da Universidade queixando-se da estética de seu sorriso. A queixa principal era o escurecimento de uma restauração na face vestibular do elemento 12 e da presença de uma lesão branca no elemento 13 (Figura 1). Durante a anamnese, a paciente relatou que a lesão existia desde o momento da irrupção do dente permanente e por ser a única lesão visível na cavidade bucal, tendo o diagnóstico de hipoplasia do esmalte, provavelmente de origem traumática. A seguir, foi feito a documentação fotográfica do caso e o exame de trans iluminação para compreensão da extensão da lesão.

Figura 1 - Aspecto inicial do caso. Mancha esbranquiçada na região incisal do dente 13 e a restauração insatisfatória no 12.

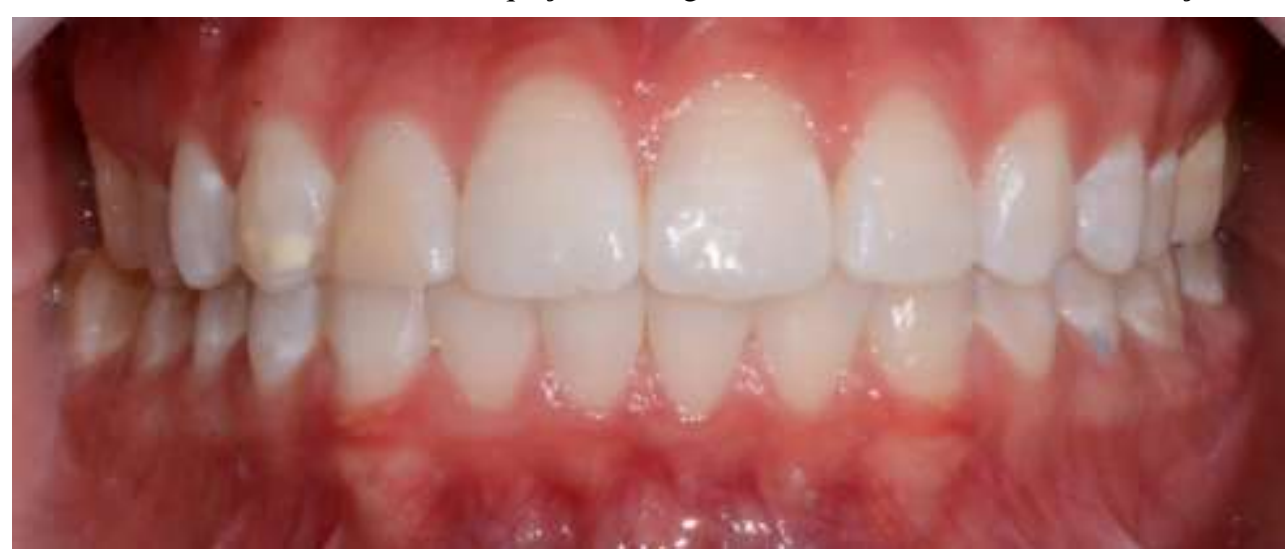

Fonte: Autores.

O tratamento proposto à paciente incluiu a substituição da restauração insatisfatória e uma associação de técnicas não invasivas para solucionar a mancha branca. Realizou-se primeiramente a limpeza dos dentes com taça de borracha associada a pedra pomes e água. Em seguida, proteção do tecido gengival com barreira fotopolimerizável (OpalDam, Ultradent, Products Inc., EUA). O gel clareador (Pola Office, SDI, Brasil) foi manipulado e aplicado na face vestibular dos dentes por 45 minutos; seguido da aplicação de gel dessensibilizante (UltraEZ, Ultradent Products Inc., EUA) por 5 minutos. O clareamento dentário foi realizado em duas sessões de clareamento de consultório com sete dias de intervalo entre cada sessão.

A continuação do tratamento se deu somente após duas semanas, o tempo de espera é necessário para que a cor dos dentes se estabilize e haja recuperação da resistência de união do esmalte. Na sessão seguinte foram realizados os procedimentos de infiltração resinosa associada à micro-abrasão, e a substituição da restauração do elemento 12. Previamente ao isolamento dos dentes anteriores maxilares com lençol de borracha, foi realizada limpeza dos dentes com pedra pomes e água.

Ao remover a camada de resina composta a ser substituída, foi observado que o elemento 12 apresentava uma lesão branca similar à do elemento 13. A sequência de procedimentos começou com a micro-abrasão. O gel abrasivo (Opalustre, 
Ultradent, Brasil) foi aplicado sobre as manchas e com auxílio de uma taça de borracha foram realizados movimentos circulares intermitentes por 20 segundos (Figura 2). Esse procedimento foi realizado três vezes, totalizando 60 segundos de aplicação.

Figura 2 - A) Lesão no dente 12 após a remoção da resina composta. B) Aplicação do microabrasivo com a taça de borracha. Aspecto logo após a micro-abrasão.
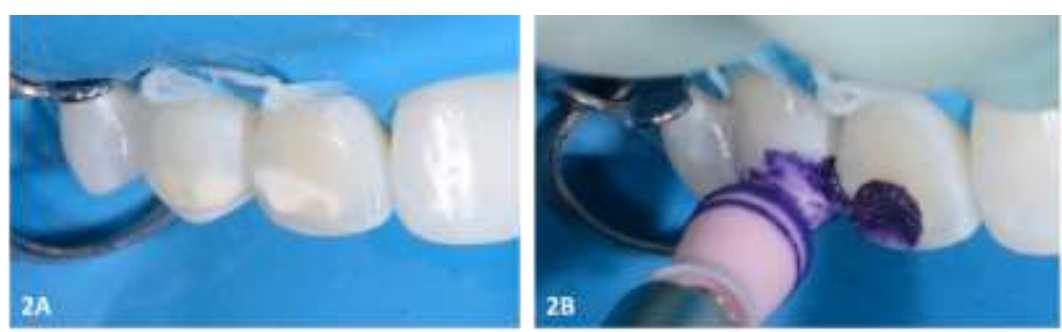

Fonte: Autores.

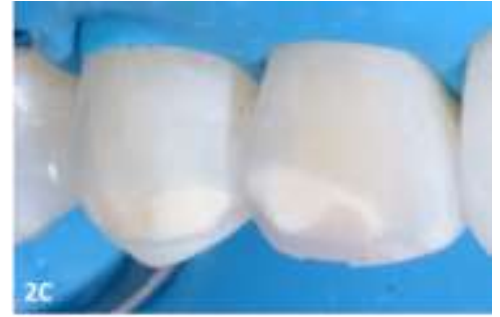

Em seguida, o procedimento de infiltração resinosa foi aplicado de acordo com as instruções do fabricante (Icon ${ }^{\mathrm{TM}}$, DMG, Alemanha). O primeiro passo consiste na aplicação do Icon Etch (ácido clorídrico 15\%) na superfície vestibular por 120 segundos. Então, os dentes foram lavados com água por 30 segundos e o Icon Dry (álcool etílico 100\%) aplicado durante 30 segundos. Após a aplicação do álcool foi observado o aspecto da mancha, que deve apresentar opacidade atenuada momentaneamente, significando um condicionamento suficiente (Figura 3A). Quando essa modificação não é perceptível, o condicionamento realizado não foi suficiente para atingir a lesão e deve ser repetido. No presente caso, esses procedimentos foram repetidos por duas vezes.

Por fim, foi realizada a aplicação Icon Infiltrant (TEGMA, aditivos e iniciadores) por 3 minutos para a penetração e assentamento do produto (Figura 3B). Após o tempo de infiltração, as faces foram fotopolimerizadas por 40 segundos e a sequência de polimento com uma borracha de acabamento (Diacomp, eve, SP, Brasil) foi efetuada (Figura 3C). Ao fim dos procedimentos conservadores, a restauração do elemento 12 foi feita com a técnica de estratificação natural. Na semana seguinte foi avaliada a cor final da restauração, e realizado os procedimentos de acabamento e polimento, e o registro fotográfico do caso finalizado. A lesão do elemento 13 não desapareceu completamente, porém houve alteração cromática e visualmente se tornou imperceptível quando hidratada (Figura 4). A paciente se mostrou completamente satisfeita com o resultado e não optou por maiores intervenções.

Figura 3 - A) Aplicação icon Etch. B) Infiltração resinosa com o Icon Infiltrant. C) Acabamento e polimento após infiltração.
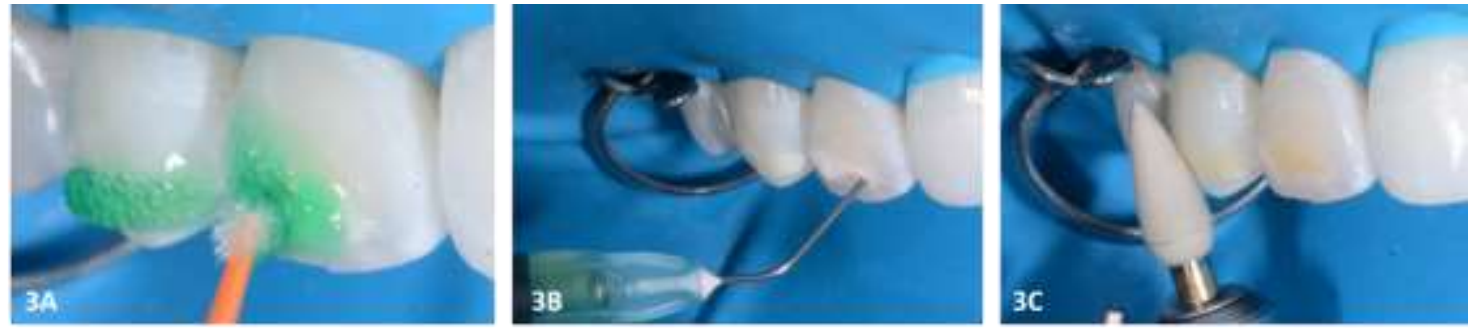

Fonte: Autores. 
Figura 4 - Aspecto final. Suavização da lesão no dente 13 e recuperação estética com a restauração no dente 12.

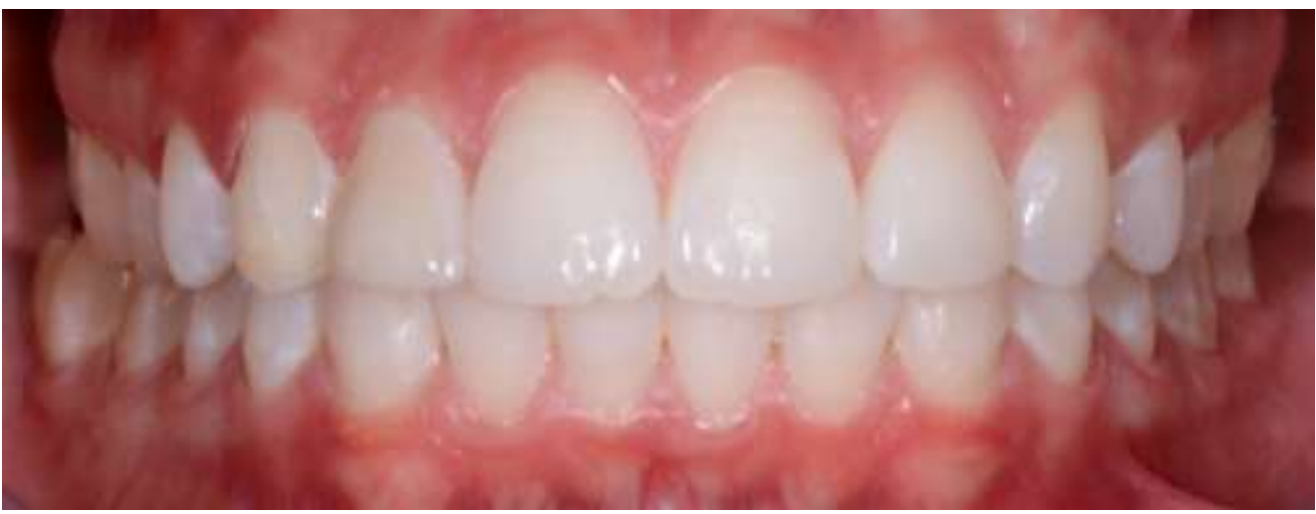

Fonte: Autores.

\section{Discussão}

O caso em questão demonstrou a eficiência da associação de técnicas não invasivas (clareamento dental, micro-abrasão e infiltração resinosa) para corrigir a queixa estética da paciente de maneira conservadora, preservando estrutura dental. O sucesso do tratamento foi possível devido ao diagnóstico correto da lesão, pois a etiologia, extensão e profundidade das lesões em esmalte influenciam seu prognóstico. Lesões superficiais, pouco volumosas e de maior translucidez, respondem melhor aos tratamentos conservadores (Denis et al., 2013). Principalmente, quando o tratamento proposto é a infiltração resinosa.

Alguns autores propuseram uma classificação para a aplicação da infiltração resinosa com dois protocolos de tratamento: a infiltração superficial, que é indicada para lesões de pequena extensão e profundidade; e a infiltração profunda, para os casos de manchas com maior volume e profundidade (Attal et al., 2014). A principal diferença entre as técnicas é a extensão da abrasão. Isto porque, quando as manchas não se encontram tão superficiais, é necessário realizar algum tipo de abrasão, podendo ser um desgaste com brocas ou mais sessões de condicionamento ácido, para que a solução infiltrante chegue até o teto da lesão (Attal et al., 2014).

A partir do exame de trans iluminação, verificamos que a técnica de infiltração superficial isolada não seria suficiente para mascarar a lesão em questão. Portanto, sugerimos o clareamento dental com o intuito de diminuir o contraste entre a mancha e o substrato sadio, que é uma prática comum quando a opção de tratamento é uma técnica não invasiva (Alverson et al., 2020; Gugnani et al., 2017; Marouane et al., 2018; Perdigão, 2020; Sundfeld et al., 2019; Sundfeld et al., 2019), seguido da infiltração resinosa profunda. No caso, indicamos a micro-abrasão como técnica erosiva, evitando o desgaste dentário por brocas (Attal et al., 2014) ou repetidas aplicações do ácido fornecido pelo fabricante (Alverson et al., 2020), como reportado por outros au tores.

Attal et al. (2014) descreveram alguns casos de infiltração resinosa profunda em que a abrasão da superfície do esmalte foi feita com brocas diamantadas ou com jateamento óxido (Attal et al., 2014). A perda de estrutura ocasionada por essas técnicas fez com que os dentes necessitassem ser restaurados após a infiltração resinosa. Como objetivo do presente caso era evitar o procedimento restaurador, optou-se por testar um protocolo "reduzido" de micro-abrasão como método erosivo para permitir que o infiltrante chegasse ao teto da lesão.

Ainda que a micro-abrasão e a infiltração resinosa utilizem o mesmo componente ácido, o ácido fluorídrico do infiltrante gera uma perda de 30 a $40 \mu \mathrm{m}$ a cada 120 s de condicionamento, enquanto a micro-abrasão gera um desgaste médio de $300 \mu \mathrm{m}$. (Pini et al., 2015; Tong et al.,1993). Esse desgaste da micro-abrasão é tempo dependente, portanto o tempo reduzido de aplicação, sugere-se que menor perda de estrutura ocorre. Desta maneira, a associação das técnicas permite uma maior profundidade de condicionamento, sem o desgaste que técnicas mais abrasivas (jateamento e brocas diamantadas) produzem.

No caso relatado, o tempo de acompanhamento foi de uma semana. Mas a literatura vem demonstrando que os resultados 
se mantêm estáveis por maiores tempos de acompanhamento (Auschill et al., 2015; Cazzolla et al., 2018; Cocco et al., 2016, Eckstein et al., 2015; Tirlet et al., 2013; Zotti et al., 2020). Embora nem todos os casos apresentem uma resolução absoluta das manchas, os relatos de sucesso com o uso da infiltração resinosa se fazem presentes na literatura para diversos tipos de lesões (Alverson et al., 2020; Brignardello-Petersen, 2017; Cavalheiro et al., 2020; Marouane et al., 2018; Muñoz et al., 2013) e demonstra ser superior a outras técnicas minimamente invasivas (Youssef et al., 2020).

Por fim, a infiltração resinosa tem se mostrado vantajosa por ser uma técnica não invasiva, de relativo baixo custo e por não necessitar de manutenção periódica. É fundamental que o clínico faça um diagnóstico correto e uma avaliação precisa da lesão para garantir o sucesso do tratamento. A variedade de protocolos e técnicas utilizadas na resolução de manchas do esmalte demostram a necessidade de realizar mais estudos clínicos controlados e randomizados para a definição de protocolos clínicos satisfatórios e orientações precisas aos profissionais.

\section{Conclusão}

A técnica de infiltração de resina quando bem indicada, parece apresentar resultados estéticos satisfatórios para a resolução de manchas a nível de esmalte nos dentes anteriores. A associação de técnicas conservadoras demonstrou sucesso para a resolução do caso em questão, que apresentava uma mancha extensa e volumosa (Figura 5A e 5B).

Apesar de demonstrar sucesso, a técnica é recente. São necessários mais estudos, principalmente com maior tempo de acompanhamento, para elucidar os resultados da técnica a longo prazo e definição de protocolos clínicos.

Figura 5 - A) Foto inicial. B) Foto final do caso realizado com 7 dias de acompanhamento.
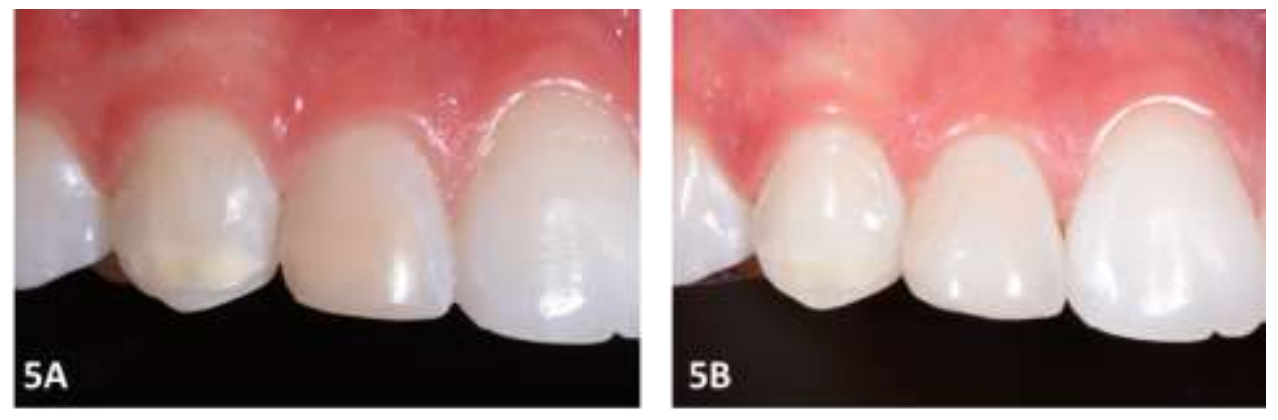

Fonte: Autores.

\section{Referências}

Abdullah, Z., \& John, J. (2016). Minimally Invasive Treatment of White Spot Lesions--A Systematic Review. Oral Health Prev Dent, 14(3), 197-205. doi:10.3290/j.ohpd.a35745.

Alverson, B. W., Capehart, K. L., Babb, C. S., \& Romero, M. F. (2020). Esthetic management of white spot lesions by using minimal intervention techniques of bleaching and resin infiltration: A clinical report. J Prosthet Dent. 10.1016/j.prosdent.2020.08.010.

Attal, J. P., Atlan, A., Denis, M., Vennat, E., \& Tirlet, G. (2014). White spots on enamel: treatment protocol by superficial or deep infiltration (part 2). Int Orthod, 12(1), 1-31. 10.1016/j.ortho.2013.12.011.

Auschill, T. M., Schmidt, K. E., \& Arweiler, N. B. (2015). Resin Infiltration for Aesthetic Improvement of Mild to Moderate Fluorosis: A Six-month Followup Case Report. Oral Health Prev Dent, 13(4), 317-322. 10.3290/j.ohpd.a32785.

Brandão, C.B., Corona, S.A.M., Torres, C.P., Côrrea-Marques, A.A., Saraiva, M.C.P., Borsatto, M.C. (2020). Efficacy of CO lasers in preventing dental caries in partially erupted first permanent molars: a randomized 18-month clinical trial. Lasers Med Sci, 35(5):1185-91. 10.1007/s10103-020-02967-7.

Brignardello-Petersen, R. (2017). Resin infiltration improves the appearance of enamel discolorations. J Am Dent Assoc, 148(4), e34. 10.1016/j.adaj.2017.02.006.

Cavalheiro, J. P., Souza, M., Duque, C. C. O., Bussaneli, D. G., Zuanon Â, C. C., \& Jeremias, F. (2020). Esthetic rehabilitation of anterior teeth with molarincisor hypomineralization and dental fluorosis: a case report. Gen Dent, 68(3), 34-39.

Cazzolla, A. P., De Franco, A. R., Lacaita, M., \& Lacarbonara, V. (2018). Efficacy of 4-year treatment of icon infiltration resin on postorthodontic white spot lesions. BMJ Case Rep, 2018. 10.1136/bcr-2018-225639. 
Ciftci, Z.Z., Hanimeli, S., Karayilmaz, H., Gungor, O.E. (2018). The efficacy of resin infiltrate on the treatment of white spot lesions and developmental opacities. Niger J Clin Pract, 21(11):1444-49. 10.4103/njcp.njcp_235_18.

Cocco, A. R., Lund, R. G., Torre, E., \& Martos, J. (2016). Treatment of Fluorosis Spots Using a Resin Infiltration Technique: 14-month Follow-up. Oper Dent, 41(4), 357-362. 10.2341/14-335-s.

Denis, M., Atlan, A., Vennat, E., Tirlet, G., \& Attal, J. P. (2013). White defects on enamel: diagnosis and anatomopathology: two essential factors for proper treatment (part 1). Int Orthod, 11(2), 139-165. 10.1016/j.ortho.2013.02.014.

Eckstein, A., Helms, H. J., \& Knösel, M. (2015). Camouflage effects following resin infiltration of postorthodontic white-spot lesions in vivo: One-year followup. Angle Orthod, 85(3), 374-380. 10.2319/050914-334.1.

Estrela, C. (2018). Metodologia Científica: Ciência, Ensino, Pesquisa. Editora Artes Médicas.

Fernandes, A.C.F., Griza, G.L., Garbin Junior, E.A., Ernica, N.M., Conci, R.A., Nadal, L. (2021). Tratamento reabilitador com reconstrução de mandíbula devido a sequela de ferimento por arma de fogo -Relato de caso. Res, Soc and Develop, 10(2), e26810212435.

Gençer, M. D. G., \& Kirzioğlu, Z. (2019). A comparison of the effectiveness of resin infiltration and microabrasion treatments applied to developmental enamel defects in color masking. Dent Mater J, 38(2), 295-302. 10.4012/dmj.2018-074.

Giray, F. E., Durhan, M. A., Haznedaroglu, E., Durmus, B., Kalyoncu, I. O., \& Tanboga, I. (2018). Resin infiltration technique and fluoride varnish on white spot lesions in children: Preliminary findings of a randomized clinical trial. Niger J Clin Pract, 21(12), 1564-1569. 10.4103/njcp.njcp_209_18.

Gu, X., Yang, L., Yang, D., Gao, Y., Duan, X., Zhu, X., \& Li, J. (2019). Esthetic improvements of postorthodontic white-spot lesions treated with resin infiltration and microabrasion: A split-mouth, randomized clinical trial. Angle Orthod, 89(3), 372-377. 10.2319/041218-274.1.

Gugnani, N., Pandit, I. K., Gupta, M., Gugnani, S., Soni, S., \& Goyal, V. (2017). Comparative evaluation of esthetic changes in nonpitted fluorosis stains when treated with resin infiltration, in-office bleaching, and combination therapies. J Esthet Restor Dent, 29(5), 317-324. 10.1111/jerd.12312.

Kau, C.H., Wang, J., Palombini, A., Abou-Kheir, N., Christou, T. (2019) Effect of fluoride dentifrices on white spot lesions during orthodontic treatment: A randomized trial. Angle Orthod, 89(3):365-71. 10.2319/051818-371.1.

Khanna, R., Chandra, A., \& Singh, R. K. (2020). Quantitative evaluation of masking effect of resin infiltration on developmental defects of enamel. Quintessence Int, 51(6), 448-455. 10.3290/j.qi.a44493.

Marouane, O., Douki, N., \& Chtioui, F. (2018). A Combined Approach for the Aesthetic Management of Stained Enamel Opacities: External Bleaching Followed by Resin Infiltration. Case Rep Dent, 2018, 1605842. 10.1155/2018/1605842.

Muñoz, M. A., Arana-Gordillo, L. A., Gomes, G. M., Gomes, O. M., Bombarda, N. H., Reis, A., \& Loguercio, A. D. (2013). Alternative esthetic management of fluorosis and hypoplasia stains: blending effect obtained with resin infiltration techniques. J Esthet Restor Dent, 25(1), 32-39. 10.1111/j.17088240.2012.00527.x.

Perdigão, J. (2020). Resin infiltration of enamel white spot lesions: An ultramorphological analysis. J Esthet Restor Dent, 32(3), 317-324. 10.1111/jerd.12550 Peters, M. C., Hopkins, A. R., Jr., Zhu, L., \& Yu, Q. (2019). Efficacy of Proximal Resin Infiltration on Caries Inhibition: Results from a 3-Year Randomized Controlled Clinical Trial. J Dent Res, 98(13), 1497-1502. 10.1177/0022034519876853.

Pereira, A. S., Shitsuka, D. M., Parreira, F. J., \& Shitsuka, R. (2018). Método Qualitativo, Quantitativo ou Quali-Quanti. In Metodologia da Pesquisa Científica. https://repositorio.ufsm.br/bitstream/handle/1/15824/Lic_Computacao_Metodologia-Pesquisa-Cientifica.pdf?sequence=1 .

Pini, N. I., Sundfeld-Neto, D., Aguiar, F. H., Sundfeld, R. H., Martins, L. R., Lovadino, J. R., \& Lima, D. A. (2015). Enamel microabrasion: An overview of clinical and scientific considerations. World J Clin Cases, 3(1), 34-41. 10.12998/wjcc.v3.i1.34.

Sundararaj, D., Venkatachalapathy, S., Tandon, A., Pereira, A. (2015). Critical evaluation of incidence and prevalence of white spot lesions during fixed orthodontic appliance treatment: A meta-analysis. J Int Soc Prev Community Dent, 5(6):433-9. 10.4103/2231-0762.167719.

Sundfeld, D., Pavani, C. C., Pavesi Pini, N. I., Machado, L. S., Schott, T. C., Bertoz, A. P. M., \& Sundfeld, R. H. (2019). Esthetic recovery of teeth presenting fluorotic enamel stains using enamel microabrasion and home-monitored dental bleaching. J Conserv Dent, 22(4), 401-405. 10.4103/jcd.Jcd_77_19.

Sundfeld, D., Pavani, C. C., Pini, N., Machado, L. S., Schott, T. C., \& Sundfeld, R. H. (2019). Enamel Microabrasion and Dental Bleaching on Teeth Presenting Severe-pitted Enamel Fluorosis: A Case Report. Oper Dent, 44(6), 566-573. 10.2341/18-116-t.

Tirlet, G., Chabouis, H. F., \& Attal, J. P. (2013). Infiltration, a new therapy for masking enamel white spots: a 19-month follow-up case series. Eur J Esthet Dent, 8(2), 180-190.

Tong, L. S., Pang, M. K., Mok, N. Y., King, N. M., \& Wei, S. H. (1993). The effects of etching, micro-abrasion, and bleaching on surface enamel. J Dent Res, 72(1), 67-71. 10.1177/00220345930720011001.

Yoo, H. K., Kim, S. H., Kim, S. I., Shin, Y. S., Shin, S. J., \& Park, J. W. (2019). Seven-year Follow-up of Resin Infiltration Treatment on Noncavitated Proximal Caries. Oper Dent, 44(1), 8-12. 10.2341/17-323-1.

Youssef, A., Farid, M., Zayed, M., Lynch, E., Alam, M.K., Kielbassa, A.M. (2020). Improving oral health: a short-term split-mouth randomized clinical trial revealing the superiority of resin infiltration over remineralization of white spot lesions. Quintessence Int, 51(9):696-709. 10.3290/j.qi.a45104.

Zotti, F., Albertini, L., Tomizioli, N., Capocasale, G., \& Albanese, M. (2020). Resin Infiltration in Dental Fluorosis Treatment-1-Year Follow-Up. Medicina (Kaunas), 57(1). 10.3390/medicina57010022. 